\title{
Through-Space Transfer of Chiral Information Mediated by a Plasmonic Nanomaterial
}

\author{
Authors: \\ Saeideh Ostovar pour ${ }^{1}$, Louise Rocks ${ }^{2}$, Karen Faulds ${ }^{2}$, Duncan Graham ${ }^{2}$, Václav Parchaňský ${ }^{3,4}$, \\ Petr Bouř ${ }^{4}$ and Ewan W. Blanch ${ }^{1,5^{*}}$

\section{Affiliations:} \\ ${ }^{I}$ Faculty of Life Sciences and Manchester Institute of Biotechnology, University of Manchester, 131 Princess Street, \\ Manchester, MI 7DN, U.K. \\ ${ }^{2}$ Centre for Molecular Nanometrology, WestCHEM, Department of Pure and Applied Chemistry, University of \\ Strathclyde, Glasgow G1 1XL, U.K. \\ ${ }^{3}$ Department of Analytical Chemistry, Institute of Chemical Technology, Technická 5, 16628 Prague, Czech \\ Republic \\ ${ }_{4}^{4}$ Institute of Organic Chemistry and Biochemistry, Academy of Sciences, Flemingovo náměstí 2, 16610 Prague, \\ Czech Republic \\ ${ }^{5}$ School of Applied Sciences, RMIT University, GPO Box 2476, Melbourne VIC 3001, Australia.
}

*Correspondence to Professor Ewan Blanch, School of Applied Sciences, RMIT University, GPO Box 2476, MelbourneVIC 3001, Australia.ewan.blanch@rmit.edu.au or e.blanch@manchester.ac.uk

\begin{abstract}
The ability to detect chirality gives stereochemically attuned nanosensors the potential to revolutionise the study of biomolecular processes. Such devices may structurally characterise the mechanisms of protein-ligand binding, the intermediates of amyloidogenic diseases and the effects of phosphorylation and glycosylation. We demonstrate that single nanoparticle plasmonic reporters, or nanotags, can enable a stereochemical response to be transmitted from a chiral analyte to an achiral benzotriazole dye molecule in the vicinity of a plasmon resonance from an achiral metallic nanostructure. The transfer of chirality was verified by the measurement of mirror image surface enhanced resonance Raman optical activity spectra for the two enantiomers of each of ribose and tryptophan. Computational modelling confirms these observations and reveals the novel chirality transfer mechanism responsible. This is the first report of colloidal metal nanoparticles in the form of single plasmonic substrates displaying an intrinsic chiral sensitivity once attached to a chiral molecule.
\end{abstract}

\section{Main Text}

Nanosculptured materials with chiral plasmonic properties (1-3) have attracted recent attention due to their ability to generate broad band circular polarization states in light as well as superchiral electromagnetic fields for ultrasensitive detection of biomolecular conformation (4). Plasmonic nanomaterials such as metallic nanoparticles exhibit strong extinction in the visible wavelength range but are achiral with no inherent chiroptical properties (1-3). It has previously been demonstrated that when biomolecules are assembled with nanomaterials, chirality from the biomolecule can be imparted to the nanomaterial, resulting in the generation of a plasmoninduced circular dichroism (CD) signal in the visible spectral region (4). This has been investigated for a limited number of chiral biomolecule/nanomaterial complexes, examples of which include DNA (5) and peptide nanotubes decorated with gold or silver nanoparticles (6-8). Optical chirality of nanostructured systems is, therefore, currently a field of active research due to potential applications in optically active devices for biomedical science, environmental 
sensing and bioterrorism detection. It has also been shown that chiral patterns can be formed by both chiral and achiral molecules (9). The phenomenon of induced chirality at a surface, observed through mirror-symmetry breaking upon molecular adsorption and transfer of handedness, has also been investigated (10). This chirality induction can occur either from chiral molecules to a non-chiral surface or from a chiral surface to non-chiral molecules. Many achiral molecules have been reported to become chiral upon adsorption on a surface (11), while breaking of the mirror symmetry of chiral molecules once adsorbed on surfaces can induce a chiral foot-print on the surface (10).

Single nanoparticle plasmonic substrates, such as hollow gold nanospheres (12), silver triangles (13), gold nanorods (14) and gold/silver silica nanoshells (15), have localized surface plasmon resonances that match the excitation wavelengths of lasers used in, for example, Raman spectroscopy. They provide a strong electromagnetic field that increases the Raman cross-section (which is a measure of the Raman signal intensity generated per molecule) giving rise to surfaceenhanced Raman scattering (SERS), and thus represent a more stable and more manageable alternative to high conjunction potentials or 'hot spots' that are characteristic of aggregated metal colloids. Additionally, they provide large scattering cross-sections that allow colorimetric detection of analytes at relatively low concentrations (16).

Colloidal suspensions of silver nanoparticles that have been functionalised with dyes, such as azo-functionalised benzotriazoles, and then coated in a silica shell, act as single plasmonic substrates that have an electronic absorption profile which can be tuned to the excitation frequency of the laser (17). These single nanoparticle plasmonic substrates can detect and provide useful information about biomolecules based on vibrational information. However, there have been no prior reports regarding their ability to have any chiroptical activity. In this paper we demonstrate the optical activity of these plasmonic substrates, in the form of resonant dye molecule-labelled nanoprobes, using Raman optical activity (ROA) spectroscopy. ROA measures the intensity difference between Raman scattering in left- and right-circular polarized light from chiral molecules (18-21). No ROA signals are detected for racemic mixtures or if a molecule possesses a plane or centre of symmetry, but spectral bands of opposing sign are detected for enantiomers (22).

Here, we report the observation of such chiroptical behaviour induced in silver silica nanotags that have an achiral structure. Furthermore, we show that chirality was induced into the achiral plasmonic surface of the substrate by enantiomeric analytes. This is a new phenomenon of chirality induction which is fundamentally different to previously reported mechanisms (23-29). We observe a symmetry breaking of surface plasmons interacting with a resonant dye molecule once they are tethered to chiral molecules. Both the silver nanoparticles which provide the surface plasmons and the dye molecules adsorbed onto the surface remain non-chiral. Once the dye molecule-labelled nanotags capture chiral molecules, in this case enantiomers of ribose and tryptophan, they become chiroptically active. The handedness of ribose and tryptophan enantiomers was transferred into the surface plasmon layer which results in opposing mirror image responses of the achiral dye reporter in the ROA spectra for the different enantiomers.

\section{Results and discussion}


Silver silica nanotags were linked with benzotriazole azo dye molecules to construct surface enhanced resonant Raman spectroscopic (SERRS) nanoprobes designed to provide a maximum enhancement at $\sim 514 \mathrm{~nm}(17)$. These dyes can act as both a resonant reporter for SERRS and a precursor for the formation of silica shells which stabilise the silver nanoparticle cores. The optical responses of the dye-labelled nanotags were first characterized $(36,37)$ and their SERRS spectra both with and without the silica nanoshell coating are shown in Figure S4 (A) and (B). Their identical SERRS profiles with strong bands originating from the dye shows that the silica shell does not interfere with the dye's SERRS signal. The corresponding surface enhanced resonant Raman optical activity (SERROA) spectra of silver nanotags with and without a silica nanoshell, Figure S4 (C) and (D), also show similar spectral features being dominated by positive bands. The positive SERROA bands resemble the parent SERRS bands with a lower signal to noise ratio which indicates in both cases that the observed bands principally originate from interaction of linear contaminants in the scattered circularly polarized light with the SERRS bands. Therefore, we conclude that these particular bands are not SERROA signals but residual artefacts, a common problem in attempts to measure the SERROA effect which necessitates careful characterisation of the interaction between the surface plasmons and circularly polarised radiation $(37,38)$. The weak features present in Figure S4 (C) and (D) between $200-1100 \mathrm{~cm}^{-1}$ indicate the noise level of these measurements.

Figure 2 displays the SERRS and SERROA spectra of L- and D-ribose covalently attached to the silver silica nanotags. The SERRS spectra of the two enantiomers of ribose have identical profiles to each other as well as to the SERRS spectra of the silver silica nanotags (Figure S4), since the resonance effect of the bound benzotriazole dye dominates the spectra. The SERS spectra of L- and D-ribose previously reported (36) are significantly different from the SERRS spectra measured for the same analytes presented here since the previous study measured direct interaction of the ribose molecules with the metal nanoparticle surfaces, which is not the case for this study. Here, the SERRS spectra shown are dominated by the characteristic bands of the benzotriazole dye molecules. Once experimental conditions were optimized the measured spectra showed good reproducibility for all spectral bands shown. Each batch of nanotags was stable for at least one month.

\section{Chiroptical response}

Crucially, the corresponding SERROA spectra presented in Figure 2 (B) and (C) for D- and Lribose exhibit a strong mirror image response and with very different features to their corresponding SERRS bands, unlike the already discussed (Figure S4) spectra obtained from the achiral silver silica nanotags. It is clear that the SERROA spectra for the two enantiomers of ribose contain both positive and negative bands, the positions of which correlate well with each other. The circular intensity difference, or ROA/Raman signal ratio, is about $4 \times 10^{-4}$ for the main SERROA bands in both cases, quantitatively verifying the mirror image response. Strong peaks appearing in the region above $1200 \mathrm{~cm}^{-1}$ in both SERROA spectra are clearly resolved from the background noise verifying the detection of the chiroptical response in the presence of L- or Dribose, which then confirms the stereochemistry of each analyte. For example, the strong +ve/ve/+ve SERROA bands at 1391, 1431 and $1450 \mathrm{~cm}^{-1}$ for L-ribose correspond to the -ve/+ve/-ve bands at 1389,1431 and $1450 \mathrm{~cm}^{-1}$ for the D-enantiomer. In addition, the strongest SERROA bands at 1616 and $1623 \mathrm{~cm}^{-1}$ for L- and D-ribose, respectively, have opposite signs as well. The differences in the band positions can be attributed to experimental noise and a limited precision 
of the CCD detector. The fact that the observed SERROA bands correspond to the SERRS bands from the benzotriazole dye rather than from the intrinsic surface enhanced Raman optical activity (SEROA) signals previously reported for L- and D-ribose (34) suggests that we are observing the effect of ribose chirality on the plasmon at the silver nanotag surface as it modifies the surface state. This chiral response is then imprinted on the SERRS spectrum of the benzotriazole dye, leading to the enantiomeric-sensitivity observed for the SERROA bands.

In order to further verify these results, the SERRS and SERROA spectra of another enantiomeric pair, L- and D-tryptophan, were also measured and are presented in Figure 3. Indeed, as before the SERRS profiles for both enantiomers are identical to those found for the benzotriazole dyetagged silver colloids. The corresponding SERROA spectra for the two enantiomers of this amino acid again display mirror image responses, in particular at 1317,1347 and $1390 \mathrm{~cm}^{-1}$, that are associated with corresponding SERRS bands from the benzotriazole nanotags. While the SERROA spectral profiles are similar for ribose and tryptophan, the intensities for ribose are significantly stronger than for tryptophan. As ROA intensities are sensitive to conformational dynamics, this suggests that the SERROA signal is also quantitatively sensitive to the greater rigidity of the cyclic ribose molecule compared to the more flexible amino acid. More mirror image bands are observed for Land D-ribose in contrast to L- and D-tryptophan within the region of $1400-1600 \mathrm{~cm}^{-1}$, such as at 1531 and $1564 \mathrm{~cm}^{-1}$, which correlates with the superior signal-to-noise ratios obtained for ribose.

\section{Computational modeling verifies our experimental results}

The experimental results are in good agreement with our modelling of the chiral response of the nanoplasmonic system once a chiral analyte is introduced. In particular, although under nonresonance conditions both ribose and some chiral conformations of the dye have distinct ROA and Raman spectra, in a complex both spectral intensities start to be dominated by the dye's vibrational bands. Our calculations verify that the chiral analyte induces a chiral response in the resonant Raman spectrum of the achiral benzotriazole dye. The SERRS and SERROA spectra simulated for the most complex model we used are plotted in Figure 4. For the dye, a smaller, arbitrarily symmetrised (of $C_{s}$ symmetry) analogue was used to avoid conformational averaging and to represent its inherently achiral character. Control computations showed that the electronic and vibrational spectral features correspond well to those of the fully functionalized benzotriazole. A ribose molecule was then placed nearby, with the centre of mass in the dye's plane, and the colloid being represented by a polarisable sphere. Spectra averaged over 64 ribose orientations were then plotted. Interestingly, both the orientational averaging and the presence of the colloid had only a minor effect on the results. Most significantly, the modelled SERROA intensities are dominated by the bands coming from the dye vibrations. The vibrational bands from ribose are invisible at this scale; they may, however, mix with those of the dye for different resonance conditions. In resonance, the simulated SERROA signal for a particular ribose enantiomer is of one sign only, as it should be for the single electronic state limit $(39,40)$. The role of the ribose in the induction of optical activity thus appears to be perturbation of the dye molecule's symmetry; or, in experiments, in the perturbation of the average spherical symmetry of the nanotag.

The observation of mirror image SERROA bands of the benzotriazole dye can thus be explained on the basis that chiral molecules, in this study L- and D-ribose or L- and D- tryptophan, once attached to the nanoprobe possess the enantioselectivity required to break the symmetric 
environment of the achiral metallic cluster. This is reported here by the SERRS response from the dye molecules which are in close proximity to the surface plasmons. In other words, induced dissymmetry in the surface plasmon interaction with the benzotriazole molecules is responsible for the observed mirror image bands in the SERROA spectra of L- and D-ribose and L- and Dtryptophan. Therefore, the induced SERROA phenomenon measured here is fundamentally different from that responsible for our previously reported SEROA spectra of L- and D-ribose (36) and the SERROA spectra of two resonant proteins myoglobin and cyctochrome c (41), since the direct interaction between the chiral molecules and the surface plasmons from metal nanoparticles in those cases was responsible for the enhancement of ROA signals. The SEROA spectral details in those previous studies also originate directly from the analyte investigated, whereas in the SERROA spectra presented in this study we observe the transfer of a chiral influence from the analyte onto the SERRS spectrum of the achiral benzotriazole dye. Chirality observed in the former systems mainly originates from dipolar interactions with chiral molecules (41). Our current observation of chiroptical behaviour monitored by dye-labelled nanotags is principally due to radiative electromagnetic coupling between the surface plasmons generated by these nanotags and the surrounding chiral molecules interacting over a long range (42). The chiral perturbation existent in the presence of achiral chromophores has induced optical activity to the metal nanoparticles and the dye, both of which act as agents enhancing the Raman signal, the former via surface plasmons, the latter via electronic resonance.

\section{A new form of chirality transfer}

The mechanism proposed as being responsible for these SERROA results can be compared to the mechanism recently reported for a class of hybrid plasmonic nanomaterials (42) sensitive to indirect adsorption of chiral molecules. Abdulrahman et al. (42) demonstrated that a chiral response was induced into the plasmonic resonance of the achiral nanostructure using circular dichroism, via measurement of electronic excitation through the radiative electromagnetic interaction between a non-absorbing isotropic chiral medium and a strongly absorbing metallic plasmon resonance. In this paper we have also observed an induced chiral response but through monitoring vibrational excitation and without the requirement of a superchiral field. In our case the plasmon resonance from the silver surface induces the SERRS signal from the tethered benzotriazole dye molecules, with the chiral analyte then interacting with that SERRS signal to generate an enantiomerically-sensitive response. The respective results from these two studies for tryptophan are revealing: Abdulrahman et al. reported no chiral response for tryptophan while Figure 3 clearly shows the chiral response evident for the same amino acid when tethered to our nanotags. Therefore, while our results involve a long-range interaction, in common with the $1 / \mathrm{d}$ dependence reported by Abdulrahman et al., our observed chiral response cannot be explained by their radiative coupling mechanism.

Cao et al. reported a through-bond mechanism of chirality transfer for CD measurements on chiral 2-amino-3-phenylpropane-1-thiol ligands directly bound to gold surfaces (43), after specifically ruling out a through space coupling as being responsible for their observations. In contrast, our modeling shows that a through bond mechanism is not responsible for our SERROA results, and our chiral analyte is around 50 bonds from the metal surface, confirming that our chirality transfer phenomenon is a fundamentally different process to that reported by Cao et al. Chirality transfer is a broad topic, and our results also appear to fundamentally differ from the electro-magnetic field (EM)-induced torsional perturbations in metamaterials consisting 
of pairs of split Cu-rings, which was reported by Liu et al. (44), and also from the transmission of chirality from single surface-bound molecules to complex chiral arrays through increasing molecular coverage on an ordered $\mathrm{Cu}(111)$ surface, as observed by Iski et al. (45).

These results clearly show that SERROA bands of opposing sign are obtained from the two enantiomers of chiral molecules and that these are signatures of a novel stereochemicallysensitive nature from the long range interactions between chiral molecules and the surface plasmons of these achiral dye-tagged nanoprobes. Comparison of this work with other recent reports of chirality transfer clarify that we have observed a distinct and new process. Extension of these studies to other analytes and nanotag designs are expected to shed further light on this exciting new chiroptical phenomenon.

\section{Methods}

\section{Synthesis of EDTA-reduced silver colloid (AgEDTA)}

Silver nanoparticles of approximately $40 \mathrm{~nm}$ diameter were synthesised according to the procedure described by Fabrikano et al. (30). Briefly, sodium hydroxide $(0.4 \mathrm{M}, 10 \mathrm{~mL})$ was injected into a boiling $1 \mathrm{~L}$ solution of EDTA $\left(1.62 \times 10^{-4} \mathrm{M}\right)$. Silver nitrate $(0.026 \mathrm{M}, 10 \mathrm{~mL})$ was added to the boiling solution in $2.5 \mathrm{~mL}$ aliquots. Following 15 minutes of continued heating, the solution was allowed to equilibrate with room temperature. Stirring was maintained throughout. The characterization of the conjugated nanoparticles is presented in the Supplementary Information.

\section{Conjugation of dye to silver nanoparticles}

Three separate replicates of nanoparticle-dye conjugates were synthesised for analysis. The trifunctional benzotriazole dye shown in Figure 1 was added to $\operatorname{AgEDTA}\left(1 \mathrm{~mL}, 1.00 \times 10^{-10} \mathrm{M}\right.$, ) to a final concentration of $10^{-7} \mathrm{M}$. Samples were agitated prior to centrifugation $(6000 \mathrm{rpm}, 20$ mins) and resuspended in $500 \mu \mathrm{L} \mathrm{dH_{2 }} \mathrm{O}$.

\section{Silica encapsulation of silver - precursor conjugates}

Silica encapsulated silver nanotags were synthesised following the method outlined in Graham et al. (17). The nanoparticle (NP) - dye conjugates were prepared to $1 \mathrm{~mL}$ with the slow addition of ethanol. Silica growth was initiated by the addition of triethylamine $(10 \mu \mathrm{L}, 1 \% \mathrm{v} / \mathrm{v}$ in ethanol) and the sequential addition of tetraethyl orthosilicate (TEOS) (10 $\mu \mathrm{L}, 4 \% \mathrm{v} / \mathrm{v}$ in ethanol) over a three hour period until the final concentration of TEOS was $5.4 \mathrm{mM}$.

\section{Functionalisation of silica encapsulated nanotags}

Based on the original NP concentration, the nanotags were functionalised with approximately $4000 \times$ molar excess of the selected enantiomer, L- or D-, of the analyte, ribose or tryptophan. This was achieved by reacting 1.21 molar equivalents of triethoxysilylpropyl isocyanate with the required enantiomer; L- or D-ribose, or L- or D-tryptophan, in $\mathrm{NaHCO}_{3}$ buffer $(0.1 \mathrm{M}$ at $\mathrm{pH}$ 9) at $4^{\circ} \mathrm{C}$ overnight. The "silanised" molecules were added to unwashed nanotags and agitated prior to centrifugation (7000 rpm, 20 mins) and resuspension $\left(500 \mu \mathrm{L} \mathrm{dH}_{2} \mathrm{O}\right)$. Due to the formation of dimers, trimers and possibly small aggregates it was difficult to determine the actual nanotag:analyte molar ratio, therefore a 1:500 ratio is quoted based on the initial nanoparticle concentration that had been used to prepare each sample. Figure 1 presents a schematic structure of the functionalised nanotags. 
All Raman and ROA spectra were measured using a ChiralRaman spectrometer (BioTools Inc., Jupiter FL, USA) configured in the backscattering geometry and operating at a wavelength of $532 \mathrm{~nm}$ and with a spectral resolution of $7 \mathrm{~cm}^{-1}$. The laser power was set to $0.20 \mathrm{~W}$, with laser power at the sample being approximately $0.10 \mathrm{~W}$, and data acquisition times ranged from $5 \mathrm{~min}$ to 2 hour.

\section{Model computations}

To investigate various factors important for the chirality transfer, we performed a number of density functional theory (DFT) computations on simplified models including the dye and chiral analyte molecules. The B3LYP (31) functional and standard 6-311++G** basis set were applied, within the Gaussian software environment (32). The universal solvation model (SMD) (33) was used to mimic the aqueous environment. Harmonic force field and frequency-dependent molecular polarizabilities needed to generate the Raman and ROA intensities (34) were generated for the excitation wavelength of $532 \mathrm{~nm}$, to simulate the near-resonance conditions. The matrix perturbation theory (35) was used to generate the spectra of a silver-dye-L-ribose complex, from the polarizability tensors obtained from Gaussian. The spectra were generated using Lorentzian profiles with full width at half height of $10 \mathrm{~cm}^{-1}$.

\section{References and Notes:}

1. Gansel, J. K. et al. Gold helix photonic metamaterial as broadband circular polarizer. Sci. Am. 325, 1513-1515 (2009).

2. Schwaneck, A. S. et al. Broken time reversal of light interaction with planar chiral nanostructures. Phys. Rev. Lett. 91, 247404 (2003).

3. Kuwata-Gonokami, M. et al. Giant optical activity in quasi-two-dimensional planar nanostructures. Phys. Rev. Lett. 95, 227401 (2005).

4. Hendry, E. et al. Ultrasensitive detection and characterization of biomolecules using superchiral fields. Nature Nanotech., 5, 783-787 (2010).

5. Slocik, J. M., Govorov, A. O \&, Naik, R. R. Plasmonic circular dichroism of peptide functionalized gold nanoparticles. Nano. Lett., 11, 701-705 (2011).

6. George, J. \& Thomas, K. G. Surface plasmon coupled circular dichroism of Au nanoparticles on peptide nanotubes. J. Am. Chem. Soc. 132, 2502-2503 (2010).

7. Shukla, N., Bartel, M. A. \& Gellman, A. J. Enantioselective separation on chiral Au nanoparticles. J. Am. Chem. Soc. 132, 8575-8580 (2010).

8. Řezanka, P., Záruba, K. \& Král, V. Supramolecular chirality of cysteine modified silver nanoparticles. Colloids Surf. A, 374, 77-83 (2011).

9. Elemans, J. A. A. W., de Cat, I., Xu, H. \& de Feyter, S. Two-dimensional chirality at liquid-solid interfaces. Chem. Soc. Rev., 38, 722-736 (2009).

10. Ernst, K. H. Supramolecular surface chirality (Springer-Verlag, Berlin, 2006), pp. 209-252.

11. Ernst, K. H. Molecular chirality in surface science. Surf. Sci., 613, 1-5 (2013).

12. Chon, H., Lee, S., Son, S., Oh, C. H. \& Choo, J. Highly sensitive immunoassay of lung cancer marker carcinoembryonic antigen using surface-enhanced Raman scattering of hollow gold nanospheres. Anal. Chem., 81, 3029-3034 (2009).

13. Camden, J. P., Dieringer, J. A., Zhao, J. \& van Duyne, R. P. Controlled plasmonic nanostructures for surface-enhanced spectroscopy and sensing. Acc. Chem. Res., 41, 1653-1661 (2008).

14. Temur, E., Boyaci, I., Tamer, U., Unsal, H. \& Aydogan, N. A highly sensitive detection platform based on surface-enhanced Raman scattering for Escherichia coli enumeration. Anal. Bioanal. Chem., 397, 15951604 (2010).

15. Jackson, J. B., Westcott, S.L., Hirsch, L. R., West, J. L. \& Halas, N. J. Controlling the surface enhanced Raman effect via the nanoshell geometry. Appl. Phys. Lett, 82, 257-259 (2003). 
16. Lin, A. W. H., Lewinski, N. A., Lee, M. \& Drezek, R. A. Reflectance spectroscopy of gold nanoshells: computational predictions and experimental measurements. J. Nanopart. Res., 8, 681-692 (2006).

17. Rocks, L., Faulds, K. \& Graham, D. Rationally designed SERS active silica coated silver nanoparticles. Chem. Comm., 47, 4415-4417 (2011).

18. Barron, L. D., Hecht, L., Blanch, E. W. \& Bell, A. F. Solution structure and dynamics of biomolecules from Raman optical activity. Prog. Biophys. Mol. Biol., 73, 1-49 (2000).

19. Freedman, T. B., Nafie, L. A. \& Keiderling, T. A. Vibrational optical activity of oligopeptides. Biopolymers, 37, 265-279 (1995).

20. Nafie, L. A. Vibrational optical activity. Appl. Spectrosc., 50, 14A-26A (1996).

21. Berova, N. et al. Circular Dichroism: Principles and application. L. D. Barron, L. Hecht, Ed. (Willey-VCH, 2000), pp.667-702.

22. Barron, L. D., Hecht, L., McColl, I. H. \& Blanch, E. W. Raman optical activity comes of age. Mol. Phys., 102, 731-744 (2004).

23. Adachi, K. et al. Chirality induction and amplification in methylene blue H-aggregates via D- and Lphenylalanine pre-adsorbed on the tungsten oxide nanocolloid surface. New Jour. Chem, 36, 21672170 (2012).

24. Angeluts, A. A. et al. Second harmonic generation upon reflection of light from the surface of a solution of mirror-asymmetric molecules: A new tool for studies of molecular chirality. Opt. Spectrosc., 87, 151156 (1999).

25. Borovkov, V. V., Lintuluoto, J. M., Fujiki, M. \& Inoue, Y. Temperature effect on supramolecular chirality induction in bis(zinc porphyrin). J. Am. Chem. Soc., 122, 4403-4407 (2000).

26. Borovkov, V. V., Yamamoto, N., Lintuluoto, J. M., Tanaka, T. \& Inoue, Y. Supramolecular chirality induction in bis(zinc porphyrin) by amino acid derivatives: Rationalization and applications of the ligand bulkiness effect. Chirality, 13, 329-335 (2001).

27. Chilaya, G. Induction of chirality in nematic phases. Rev. Phys. Appl., 16, 193-208 (1981).

28. Chavan, S. P. \& Khatod, H. S. Enantioselective synthesis of the essential oil and pheromonal component ar-himachalene by a chiral pool and chirality induction approach. Tetrahedron: Asymmetry, 23, 14101415 (2012).

29. Fireman-Shoresh, S., Marx, S. \& Avnir, D. Induction and detection of chirality in doped sol-gel materials: NMR and circular dichroism studies. J. Mater. Chem., 17, 536-544 (2007).

30. Fabrikanos, V. A., Athanassiou, S. \& Lieser, K. Dastelung stabiler hydrosole von gold und silber durch reduktion mit athylendiamintetraessingsaure. Z-Naturforsch. B, 612-616 (1963).

31. Becke, A. D. Density-functional thermochemistry. III. The role of exact exchange. J. Chem. Phys., 98, 5648-5652 (1993).

32. Frisch, M. J. et al., Gaussian 09, Revision D01. 2009, Gaussian, Inc.: Wallingford CT.

33. Marenich, A. V., Cramer, C. J. \& Truhlar, D. G. Universal solvation model based on solute electron density and on a continuum model of the solvent defined by the bulk dielectric constant and atomic surface tensions. J. Phys. Chem. B, 113, 6378-6396 (2009).

34. Barron, L. D. Molecular light scattering and optical activity. (Cambridge: Cambridge University Press, 2004).

35. Novák, V., Šebestík, J. \& Bouř, P. Theoretical modeling of the surface-enhanced Raman optical activity. J. Chem. Theory Comput., 8, 1714-1720 (2012).

36. Ostovar pour, S., Bell, S. E. J. \& Blanch, E. W. Use of a hydrogel polymer for reproducible surface enhanced Raman optical activity (SEROA). Chem. Comm., 47, 4754-4756 (2011).

37. Abdali, S. \& Blanch, E. W. Surface enhanced Raman optical activity (SEROA). Chem. Soc. Rev., 37, 980992 (2008).

38. Etchegoin, P. G., Galloway, C. \& Le Ru, E. C. Polarization-dependent effects in surface-enhanced Raman scattering (SERS). Phys. Chem. Chem. Phys., 8, 2624-2628 (2006).

39. Nafie, L. A. Theory of resonance Raman optical activity: The single electronic state limit. Chem. Phys., 205, 309-322 (1996).

40. Nafie, L. A. Vibrational optical activity: Principles and applications. (Chichester: John Wiley \& Sons, 2011). 
41. Abdali, S., Johannessen, C., Nygaard, K. \& Nørbygaard, T. Resonance surface enhanced Raman optical activity of myoglobin as a result of optimized resonance surface enhanced Raman scattering conditions. J. Phys.-Condens. Mat., 19, 285205 (2007).

42. Abdulrahman, N. A. et al., Induced chirality through electromagnetic coupling between chiral molecular layers and plasmonic nanostructures. Nano. Lett., 12, 977-983 (2012).

43. Cao, T. et al., A comparison of the chiral counterion, solvent, and ligand used to induce a chiroptical response from Au25 nanoclusters. Nanoscale, 5, 7589-7595 (2013).

44. Liu, M. et al., Spontaneous chiral symmetry breaking in metamaterials. Nature Comm. | 5:4441 | DOI: 10.1038/ncomms5441 (2014).

45. Iski, E. V., Tierney. H. L., Jewell, A. D. \& Sykes, E. C. H. Spontaneous transmission of chirality through multiple length scales. Chem. Eur. J., 17, 7205-7212 (2011).

Acknowledgments: This work was supported by the UK Engineering and Physical Sciences Research Council (EP/P504325/1 and GR/S75727/01), Royal Society of Chemistry Analytical Sciences Division Studentship Scheme (GR/T05554/01), Academy of Sciences (M200551205), Grant Agency (P208/11/0105, 13-03978S) and Ministry of Education (LH11033) of the Czech Republic. The research data associated with this paper will become available at the following link from July 2015: https://pure.strath.ac.uk/portal/en/projects/doctoral-training-grant-2008-ra4346\%2827114569-e902-4216-90d1-a745eb78bdb1\%29.html. The authors wish to thank Ewen Smith, Malcolm Kadodwala, Euan Hendry, Christian Johannessen, Simon Webb and Andrew Doig for helpful discussions, and DG thanks the Royal Society for support from a Wolfson research merit award.

Author Contributions: EB directed the project, DG and KF led the nanoparticle preparation and characterisation, PB led the computational modelling, SOP performed the SERROA studies, SOP and LR both performed SERS and characterisation experiments and VP performed computational modelling. All authors contributed to preparing the manuscript, with EB and SOP being the main authors. 
Fig. 1. Schematic of the silver nanotag, including the nanoparticle core and adsorbed benzotriazole azo reporter molecules, surrounding silica shell and attached chiral analyte.

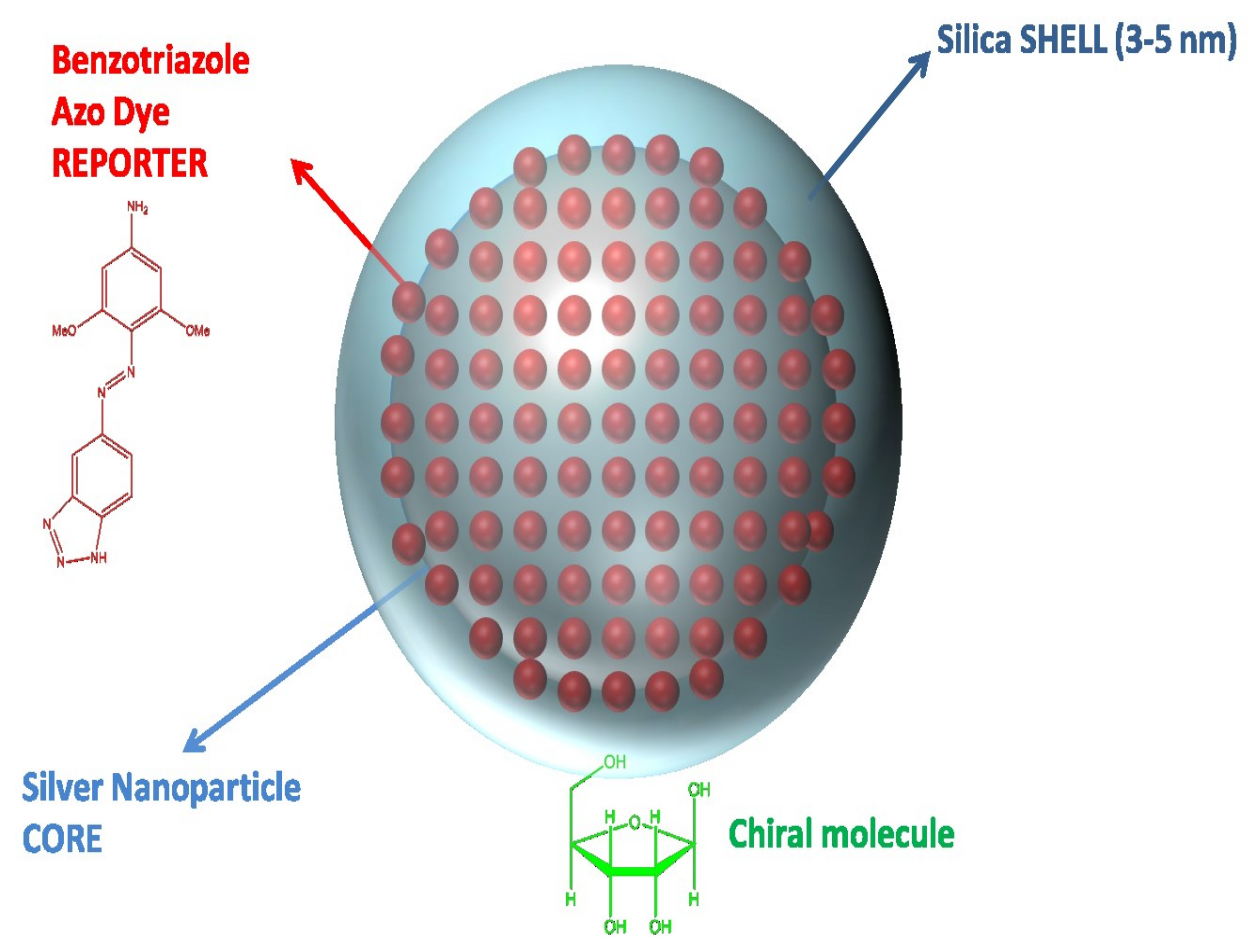


Fig. 2. SERRS spectra of D- and L-ribose that attached to silver silica nanotag (A), SERROA spectra of D- and L-ribose for replicate experiments (B) and (C) illustrating the level of reproducibility, with data collection time of $35 \mathrm{~min}$ and laser power at source $0.20 \mathrm{~W}$. Identical SERRS spectra are observed for D- and L-ribose as SERRS is blind to chirality, while mirror image SERROA bands are measured for the two enantiomers signifying the chiroptical sensitivity of these nanotags.

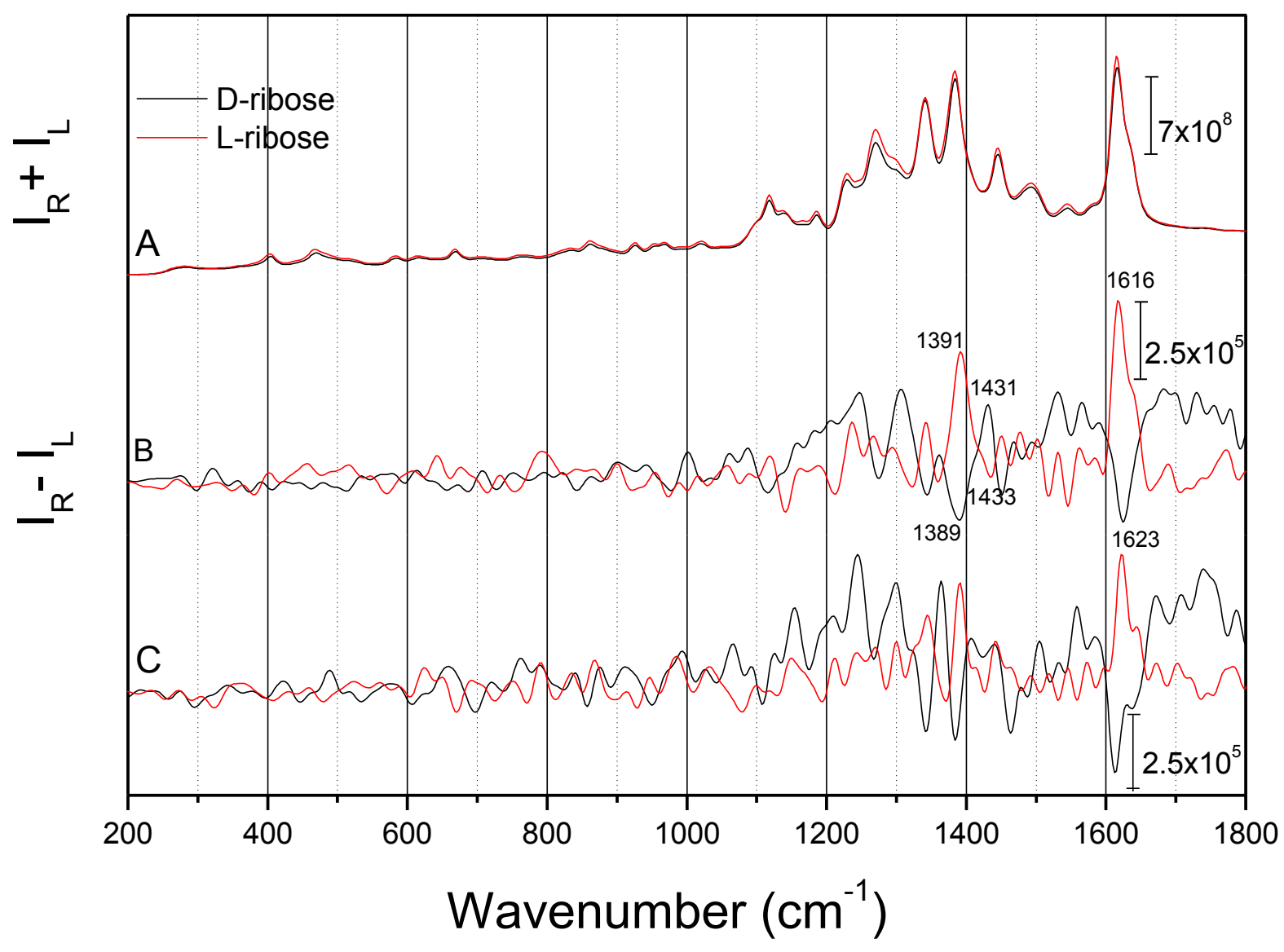


Fig. 3. SERRS spectra of D- and L-tryptophan that attached to silver silica nanotag (A), SERROA spectra of D- and L-tryptophan (B), data collection time of $35 \mathrm{~min}$ and laser power at source $0.20 \mathrm{~W}$. The nanotags also show strong chiroptical responses in the SERROA spectra of L- and D-tryptophan.

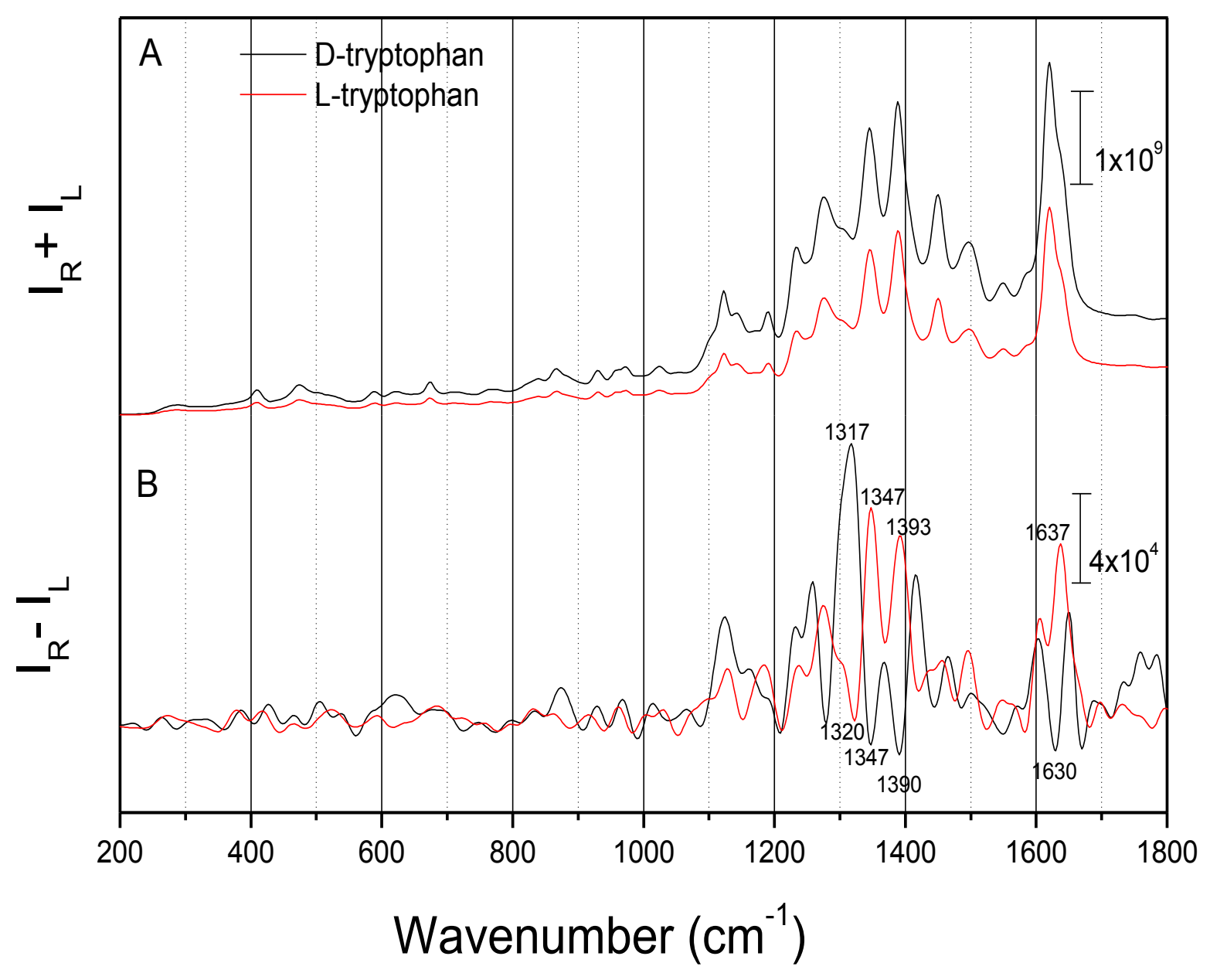


Fig. 4. A model system and its Raman and ROA spectra, generated by the matrix perturbation theory. The colloid is represented by a polarizable sphere $\left(\alpha=10^{5}\right)$ as shown in Panel $\mathrm{A}$, for the simplified dye and ribose molecule the ROA tensors were calculated at the B3LYP/6-31G* (SMD) level at the excitation frequency of $532 \mathrm{~nm}$, and averaged over ribose rotation around its mass center. The modelled SERRS spectra for D- and L-ribose overlap perfectly (Panel B), while the DFT model verifies that the stereochemically-sensitive SERROA bands (Panel C) originate from the vibrations of the benzotriazole dye molecule.
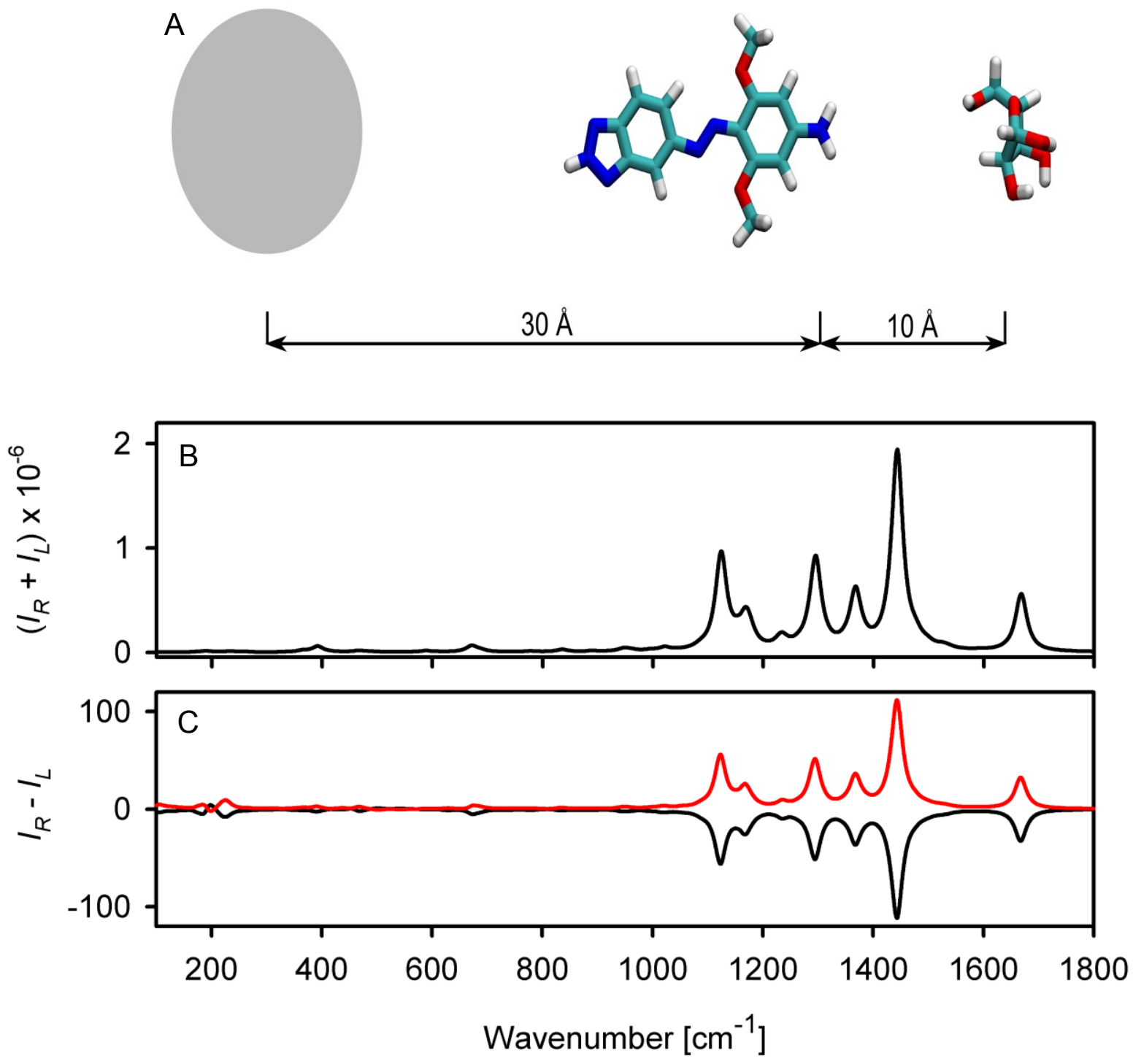